\title{
Sodium stibogluconate cardiotoxicity and safety of generics
}

S. Rijal ${ }^{1}$, F. Chappuis ${ }^{2}$, R. Singh ${ }^{1}$, M. Boelaert ${ }^{3}$, L. Loutan $^{2}$ and S. Koirala $^{1}{ }^{1}$ B. P. Koirala Institute of Health Sciences, Dharan, Nepal; ${ }^{2}$ Travel and Migration Medicine Unit, Geneva University Hospital, 1211 Geneva 14, Switzerland; ${ }^{3}$ Department of Public Health, Prince Leopold Institute of Tropical Medicine, Antwerp, Belgium

\begin{abstract}
Between April 9 and May 5 2000, an outbreak of fatal cardiotoxicity occurred in Nepal amongst visceral leishmaniasis patients treated with a recently introduced batch of generic sodium stibogluconate (SSG) from GL Pharmaceuticals, Calcutta, India. Eight (36\%) of 23 patients treated with this batch died, and in $5(23 \%)$ death was attributed to the cardiotoxicity of the drug. This contrasts with the low total death rate $(3.2 \%)$ and death rate due to cardiotoxicity $(0.8 \%)$ observed among 252 patients treated between August 1999 and December 2001 with generic SSG from Albert David Ltd, Calcutta, India. These data show that every batch of generic SSG should be subject to rigorous quality control prior to use.
\end{abstract}

Keywords: visceral leishmaniasis, chemotherapy, sodium stibogluconate, generic drugs, cardiotoxicity, Nepal

\section{Introduction}

Visceral leishmaniasis (VL: kala-azar) remains highly endemic in many poor rural regions of India and Nepal. First-line treatment for visceral leishmaniasis in Nepal is sodium stibogluconate (SSG) $20 \mathrm{mg} / \mathrm{kg} / \mathrm{d}$ i.m. for $30 \mathrm{~d}$, as recommended by WHO. In Nepal, the Epidemiology and Disease Control Division (EDCD), Ministry of Health, distributes SSG to all hospitals managing VL patients.

As a high level of resistance to antimonials is currently being observed in the neighbouring Indian state of Bihar (Sundar et al., 2000), we monitored the efficacy of SSG therapy at the B. P. Koirala Institute of Health Sciences (BPKIHS), a tertiary care centre located in Dharan, Nepal, from August 1999. For several years, the source of SSG was an Indian generic supplier (Albert David Ltd, Calcutta, India), and this drug, also called sodium antimony gluconate (SAG), has been used at BPKIHS without significant problems of toxicity or lack of efficacy. From 2 April 2000 onwards, the EDCD central pharmacy supplied generic SSG from another Indian manufacturer, GL Pharmaceuticals, Calcutta, India. At the beginning of May 2000, physicians at BPKIHS were alerted by an increase in the case-fatality rate in VL patients. The recently introduced brand of SSG was highly suspected as the potential cause of the increased mortality and it was discontinued on 7 May 2000. From that date onwards, VL patients were again treated with generic SSG manufactured by Albert David Ltd. We report the investigation of this increased mortality.

\section{Methods}

We carefully reviewed the files of all the 23 patients treated with SSG from GL Pharmaceuticals. Moreover, we also analysed the outcome for VL admitted to BPKIHS since August 1999, and reviewed the causes of death reported.

\section{Results}

Between August 1999 and December 2001, BPKIHS admitted 275 patients with parasitologically confirmed VL for treatment with SSG. All patients were admitted for at least 1 week and $42 \%$ were admitted for the full duration of treatment. Ninety-five percent of patients had a check-up performed at 1 month after completion of the drug course. Of the 23 VL patients who received 5 or more doses of SSG from GL Pharmaceuticals, $8(36.4 \%)$ died, including 5 $(22.7 \%)$ with either ECG-proven (ventricular tachycardia or fibrillation) or high clinical suspicion (cardiac

Address for correspondence: Dr F. Chappuis, Travel and Migration Medicine Unit, Geneva University Hospital, rue Micheli-du-Crest 24, 1211 Geneva 14, Switzerland; phone +41223729620, fax +41223729626,

e-mail francois.chappuis@hcuge.ch arrest) of cardiotoxicity. These 5 patients received a median of 27 doses of SSG (range 8-34). Of the other 3 patients $(13.6 \%)$ who died, the cause of death was bacterial meningitis in 1 and sepsis with bleeding in the other 2. In contrast, during a 2.5-year period, of the 252 patients treated with SSG from Albert David Ltd, only $8(3.2 \%)$ had a fatal outcome (Figure). Of the 8 deaths, $2(0.8 \%)$ were attributed to cardiotoxicity. The relative risk of death in patients treated with SSG from GL Pharmaceuticals compared with patients treated with SSG from Albert David Ltd was 11.0 (95\% CI $4.5-26.5)$.

\section{Discussion}

The risk of fatal complications in patients treated with SSG for leishmaniasis should not be underestimated. Whereas SSG is considered safe at the WHO recommended dosages by Berman (1988), this mainly refers to patients treated for cutaneous or mucocutaneous leishmaniasis. In Indian VL patients, death due to cardiotoxicity was recently reported in $5.9 \%$ of patients (Sundar et al., 2000). Visceral leishmaniasis patients frequently present with an altered general condition including malnutrition and superimposed bacterial infections. Cardiotoxicity is more likely in these patients, possibly because of concomitant electrolyte disturbances, micronutrient or vitamin deficiencies. An outbreak of cardiotoxicity has already been reported in India where 3 of 8 patients treated with generic SSG died due to an excessively high osmolarity (Sundar et al., 1998). Unfortunately, analysis of the content of the suspected batch of SSG from GL Pharmaceuticals used at BPKIHS could not be performed because all remaining vials were rapidly called back and destroyed by the EDCD in Kathmandu to prevent its further use in the endemic region. The increased mortality observed at BPKIHS in April and May 2000 was also observed concomitantly in several other hospitals in Nepal. Interestingly, a similar problem involving the same SSG manufacturer seemed to have occurred also in India during the same period as the 10 June 2000 edition of an Indian daily newspaper 'The Statesman' published a front-page article entitled 'The return of the killer injection' (Chakraborty, 2000)

The production of SSG is a difficult process and a significant proportion produced has commonly to be discarded because it is of insufficient quality. This might explain partly the high cost of branded antimonials (US\$150-200 per patient for a full drug course), and gives food for thought as to the origin of toxic generic batches. Generic SSG was advocated as a solution to the drug access problem in developing countries, because of its better affordability (US $\$ 13-16$ per patient per course). In that regard, recent studies comparing the generic SSG (SAG) from Albert David Ltd and the branded SSG (Pentostam ${ }^{\circledR}$ from Glaxo-Wellcome, London, UK) performed in Kenya, Sudan, and 
$\leftrightarrow$ Period of suspect batch use $\quad$ Patients treated with SSG $\square$ All deaths $\square$ Deaths due to cardiotoxicity

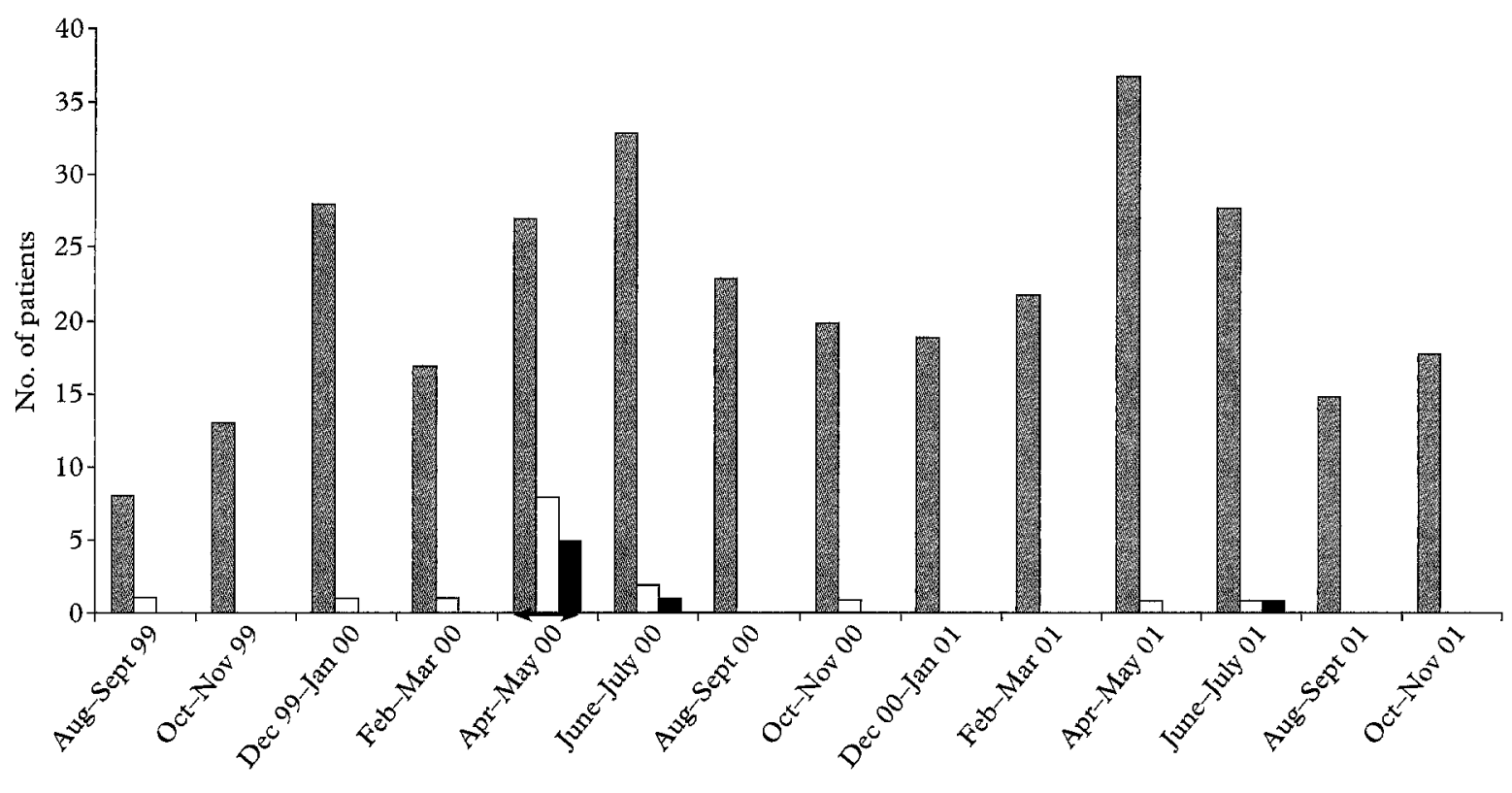

Time period

Figure. Number of patients, total deaths, and deaths due to cardiotoxicity amongst visceral leishmaniasis patients treated with generic sodium stibogluconate at the B. P. Koirala Institute of Health Sciences, Dharan, Nepal from August 1999 to December 2001 .

Ethiopia showed equivalent efficacy and toxicity (Veeken et al., 2000; Moore et al., 2001; Ritmeijer et al., 2001). The use and distribution of cheaper generic antimonials should thus be strongly promoted but this should be done with appropriate assurance of safety and efficacy. We believe that generic SSG should be widely used in VL-endemic countries but that rigorous quality controls should be performed on every batch to prevent outbreaks of fatal complications as reported here. For example, independent quality control of every single batch of SSG from Albert David Ltd is currently being implemented by the International Dispensary Association, Amsterdam, The Netherlands before distribution to East African countries. Also, the use of lower total doses of SSG by using combination therapies (e.g. SSG with paromomycin) might efficiently decrease its dose-dependent cardiotoxicity.

\section{References}

Berman, J. D. (1988). Chemotherapy for leishmaniasis: biochemical mechanisms, clinical efficacy, and future strategies. Review of Infectious Diseases, 10, 560-586.

Chakraborty, A. (2000). Return of the killer injection. The Statesman, 10 June, pp. 1 and 5.

Moore, E., O'Flaherty, D., Heuvelmans, H., Seaman, J., Veeken, H., de Wit, S. \& Davidson, R. (2001). Comparison of generic and proprietary sodium stibogluconate for the treatment of visceral leishmaniasis in Kenya. Bulletin of the World Health Organization, 79, 388-393.

Ritmeijer, K., Veeken, H., Melaku, Y., Leal, G., Amsalu, R., Seaman, J. \& Davidson, R. N. (2001). Ethiopian visceral leishmaniasis: generic and proprietary sodium stibogluconate are equivalent; HIV co-infected patients have a poor outcome. Transactions of the Royal Society of Tropical Medicine and Hygiene, 95, 668-672.

Sundar, S., Sinha, P. R., Agrawal, N. K., Srivastava, R., Rainey, P. M., Berman, J. D., Murray, H. W. \& Singh, V. P. (1998). A cluster of cases of severe cardiotoxicity among kala-azar patients treated with a high-osmolarity lot of sodium antimony gluconate. American foumal of Tropical Medicine and Hygiene, 59, 139-143.

Sundar, S., More, D. K., Singh, M. K., Singh, V. P., Sharma, S., Makharia, A., Kumar, P. C. K. \& Murray, H. (2000). Failure of pentavalent antimony in visceral leishmaniasis in India: report from the center of the Indian epidemic. Clinical Infectious Diseases, 31, 1104-1107.

Veeken, H., Ritmeijer, K., Seaman, J. \& Davidson, R. (2000). A randomized comparison of branded sodium stibogluconate and generic sodium stibogluconate for the treatment of visceral leishmaniasis under field conditions in Sudan. Tropical Medicine and International Health, 5, 312-317.

Received 12 September 2002; revised 28 March 2003; accepted for publication 3 April 2003 